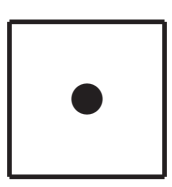

\title{
Small Phenomena, Big Implications
}

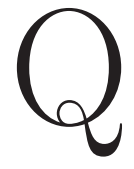

UANTUM Information Science (QIS) is the merger of quantum mechanics and information science. These are rich fields that few study in great detail. The three most important QIS concepts that underlie quantum technologies are: uncertainty, entanglement, and superposition. After introducing the three technical concepts, we outline the highest-level policy challenges in quantum technologies.

This chapter is written for people who neither have nor want a background in quantum physics. It is written at a high level, and thus necessarily omits nuance in favor of basic comprehension. After reading this short summary, the reader then has a choice: continue on where we present quantum technologies from a functional perspective, or you can turn to the appendixes of this book (p. 471), where the three concepts receive a much deeper treatment. ${ }^{1}$

\subsection{Uncertainty}

The concept of uncertainty is the core concept of quantum mechanics. Simply put, uncertainty means that it is physically impossible to know everything about anything. More specifically, uncertainty

\footnotetext{
${ }^{1}$ The Appendixes explore the how and sometimes the why of quantum technologies. Readers who have to make key decisions surrounding quantum technologies, such as the decision to invest money or to make predictions surrounding the technology, should first invest in understanding the basics as presented in the last part of this book.
} 
means that it is impossible to know specific things about the physical world with total accuracy.

We all manage to get by with significant uncertainty in society. For example, it is uncertain how many dollars there were in the US economy at the stroke of midnight in Washington, D.C. on January 1,2021 . But this is a different kind of uncertainty than we deal with in quantum physics. In principle we could obtain the bank records of every US corporation and individual, go house-to-house and count all of the cash, go through every hotel and look at all of the loose change that had fallen into every sofa, and with all that information come up with the size of the money supply. Making that measurement with high accuracy would depend upon having a precise time at which the measurements were being made and an army of auditors to make it. With enough information, one could be certain about the state of the economy.

Quantum uncertainty is different than economic uncertainty because it is typically described in terms of two quantities that are antagonistic: the more accurately one is measured, the less accurate the other. Physicists use the word complementarity to describe this antagonistic quality. One explanation for this is that measurement is a physical act, and thus measuring an object requires interacting with that object, which influences its quantum state.

Physicists will not like this simple explanation of uncertainty, but it is good enough for our purposes.

\subsection{Entanglement}

Entangled particles are somehow linked on the quantum level, even though they are separated in physical space and have no known way of communicating with each other. When two particles are entangled, a measurement made on the first may be correlated with a measurement made on the second. Albert Einstein called entanglement "spooky action at a distance" because it means the measurement of one particle somehow effects another distant one. Yet there is no transmission of information from particle to the other: the two particles are simply linked in some "spooky" way.

Entanglement is a quantum phenomenon with no classical analogue. One way to think about entanglement is that particles that are entangled are part of the same system. When one measures one part of the system, for instance the polarization of a photon (the orienta- 
tion of a light wave), the measurement of that photon's polarization reveals something about the other, entangled photon.

Two entangled photons can be produced by shining an infrared laser on a lithium niobate crystal: sometimes a single photon will appear to be "split" into two photons, each with half the frequency of the first, traveling in different directions. ${ }^{2}$ The two resulting photons are entangled: if the polarization of one is measured to be horizontal, the polarization of the second will be measured to be vertical.

Entanglement as a technique is used in all three classes of quantum technology discussed in this book. In metrology and sensing, an entangled photon pair can be used such that one photon illuminates an object while the linked photon is measured. An example comes from still-in-development radar and navigation systems, where the illuminating photon is focused on airplanes in the sky or on underwater hazards. By comparing measurements of the reflected photon with the photon that was retained, it may be possible to detect an adversary's jet or an underwater mine that could not be sensed with a single photon. In quantum computing, entanglement is used to coordinate ensembles of "qubits," the quantum version of classical computing bits. In quantum communications, entanglement can be used to ensure the distribution of secure encryption keys.

\subsection{Superposition}

Because of uncertainty, quantum mechanics is fundamentally a probabilistic view of reality: some outcomes are more probable, and some are less probable. Superposition is the word that quantum physicists use to describe the state of a quantum system before we measure it and learn the outcome of a specific experiment or manipulation.

One way to think about superposition is to consider the state of a coin at an athletic event when a referee flips it up into the air and catches it - but before the coin's state is revealed (see Figure 1.1). The coin could be heads-up or it could be tails-up. Until the referee and the players know, either outcome is equally possible.

A coin toss isn't actually quantum superposition, however, because there are ways that the outside universe could know about the coin's state even before it is revealed by the referee. For example, a spectator with a telescope and a high-speed camera might have recorded the position of the coin at the exact instant that it was

${ }^{2}$ Prabhakar et al., "Two-Photon Quantum Interference and Entanglement at 2.1 Mm" (2020). 


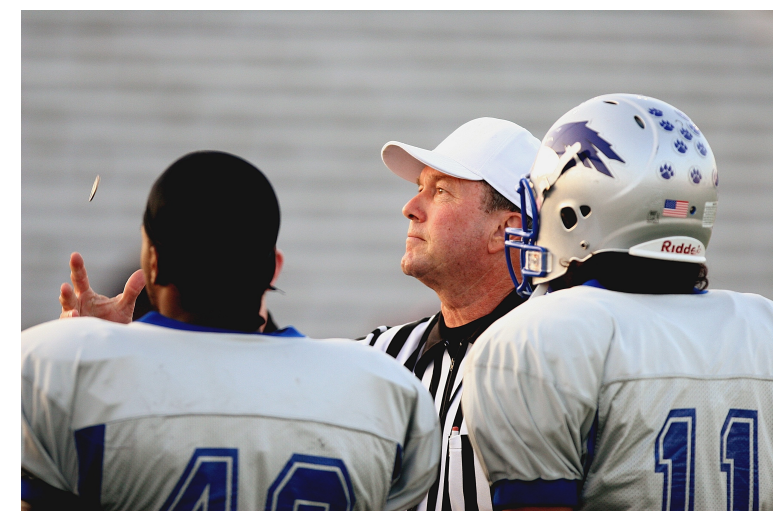

Figure 1.1. Superposition is like the state of a coin in an official coin toss at an athletic event after the coin has stopped spinning but before it has been revealed. Coin toss photo by Keith Johnston from Pixabay.

covered by the referee's hand. Or the referee may be able to feel the position of the coin, and somehow telegraph that knowledge.

A quantum-random coin toss would be truly random and invulnerable to the observation tricks of the spectator or corrupt referee. But the process would impose constraints on the referee. The referee would have to isolate the quantum coin toss from the noise and energy of the universe, lest inference affect the randomness. Instead of using a coin, hands, and eyes, the referee might use a particle of uranium and two Geiger counters entombed in a special, sealed room. As the uranium naturally and randomly emits single quanta of radiation, a Geiger counter clicks. One Geiger counter is labeled "heads," the other "tails." The referee turns on the two Geiger counters at precisely the same time and then notes which counter clicked first.

In this example, the uranium, the referee, and the Geiger counters are in a superposition of two states: one where the heads Geiger counter clicks first, the other where tails clicks first. Until the referee leaves the room and interacts with the rest of the universe, either outcome is equally possible, even many seconds (or even hours) after the Geiger counters were first switched on.

Of course, we would have a hard time building a room that would truly isolate the referee from the rest of the universe, and so in practice we do not experience superposition in our daily lives. Nevertheless, supposition is a critical component of many QIS-based instru- 
ments. Superposition allows quantum computers to compute directly with quantum probabilities. QIS engineers use the term coherence to describe such a system that is in the probabilistic superposition state, before it has interacted with the rest of the universe.

Most QIS systems today require cooling the active components to near absolute zero, in order to shield the quantum state from thermal noise and maintain coherence. QIS devices may also be surrounded by a curious material called mu-metal which shields from magnetic fields.

Because the quantum systems that we use are typically based on the interaction of photons, electrons, and occasionally entire atoms, they don't require shielding from gravity. This is a good thing, because we (currently) don't know how to shield something from gravity. At the same time, there are some quantum sensing devices that use our inability to shield from gravity as a way of measuring minor changes in gravity, which can be used to detect underground mineral deposits and even objects. With even more sensitive devices we can detect gravity waves, although such waves are detected indirectly by their impact on the fabric of space-time, as we shall see in the next chapter.

The exquisite sensitivity of quantum states is both the source of quantum technologies' utility and a challenge to technology development. Quantum states' fragility make them sensitive to small perturbations, a fantastic quality for measuring subtle phenomena, such as the precise contours of the Earth's magnetic field. Yet, that same fragility is a barrier to quantum computing, where information processing requires maintaining quantum states free of environmental perturbations.

\subsection{Conclusion}

From this intentionally brief introduction to quantum effects, we turn to covering the most exciting developments in quantum sensing, quantum computing, and quantum communication. 
